\title{
A Noiseless Quantum Optical Memory at Room Temperature
}

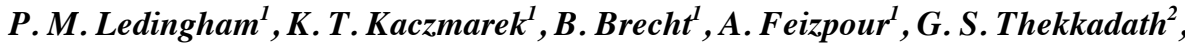 \\ S.E.Thomas ${ }^{1,3}$, J. H.D. Munns ${ }^{1,3}$, D. J.Saunders ${ }^{1}$, J. Nunn ${ }^{1}$, I. A. Walmsley ${ }^{1}$ \\ 1. Clarendon Laboratory, University of Oxford, Parks Road, Oxford OX1 3PU, UK. \\ 2. National Research Council of Canada, Ottawa, Canada. \\ 3. QOLS, Blackett Laboratory, Imperial College London, London SW7 2BW, UK.
}

A quantum optical memory (QM) is a device that can store and release quantum states of light on demand. Such a device is capable of synchronising probabilistic events, for example, locally synchronising nondeterministic photon sources for the generation of multi-photon states, or successful quantum gate operations within a quantum computational architecture [1], as well as for globally synchronising the generation of entanglement over long distances within the context of a quantum repeater [2]. Desirable attributes for a QM to be useful for these computational and communicational tasks include high end-to-end transmission (including storage and retrieval efficiency), large storage-time-bandwidth product, room temperature operation for scalability and, of utmost importance, noise free performance for true quantum operation.

Impressive realisations of QMs have materialised based on optical transitions in atomic systems [3-7]. However, several issues remain that prevent these devices from being used within large-scale networks. These include: elaborate cold atom [3,4] or cryogenic [5] experimental setups, complex preparation of the atomic system [5], additional loss through filtering required for noise suppression, and noise photons being induced in the same frequency, spatial or temporal mode as the output thus reducing the quality of the QM readout [3-7].

Here we present a new QM protocol that addresses the above issues, the quantum ladder memory (QLAD). This protocol is based on a two-photon 'ladder' transition between the $6 \mathrm{~S}_{1 / 2}$ ground state and $6 \mathrm{D}_{5 / 2}$ excited state of a caesium (Cs) ensemble at room temperature (see Fig. 1a). We characterise the memory with weak coherent states, storing GHz-band pulses with $\eta=22 \%$ storage and retrieval efficiency and a characteristic storage time of $\tau=5.3 \mathrm{~ns}$. We measure the noise at the output to be $8 \times 10^{-6}$ photons per pulse, giving rise to a noise-to-efficiency ratio of $3.6 \times 10^{-5}$, three orders of magnitude better than any memory to date. We generate GHz-band heralded single photons from a spontaneous parametric down conversion source that are matched to the QLAD, coincidence traces seen in Fig. 1b. A heralded second-order autocorrelation function of $\mathrm{g}^{(2)}=0.02 \pm 0.005$ is measured for the input photon, and true quantum operation is confirmed by storing and retrieving this photon with $\mathrm{g}^{(2)}=0.03 \pm 0.01$ for a $3.5 \mathrm{~ns}$ storage time. This result is the lowest $\mathrm{g}^{(2)}$ on the output of an on-demand memory to date, and represents a significant step toward large-scale quantum technologies.

(a)
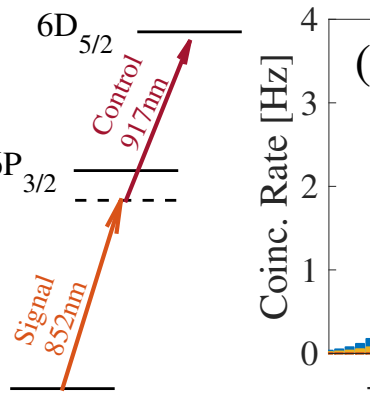

Fig. 1 (a) QLAD protocol in Cs level structure. (b) Coincidence histogram of storage and retrieval of heralded single photons. The blue (yellow) histogram represents the memory off (on), labelled Input (Output). (c) Log scale with Output and Noise (dark red histogram).

[1] J. Nunn, N. K. Langford, W. S. Kolthammer, T. F. M. Champion, M. R. Sprague, P. S. Michelberger, X.-M. Jin, D. G. England, and I. A. Walmsley "Enhancing Multiphoton Rates with Quantum Memories," Phys. Rev. Lett. 110, 133601 (2013).

[2] N. Sangouard, C. Simon, H. de Riedmatten, and N. Gisin "Quantum repeaters based on atomic ensembles and linear optics," Rev. Mod. Phys. 83, 33 (2011)

[3] T. Chanelière, D. N. Matsukevich, S. D. Jenkins, S.-Y. Lan, T. A. B. Kennedy, and A. Kuzmich "Storage and retrieval of single photons transmitted between remote quantum memories," Nature 438, 833 (2005)

[4] D.-S. Ding, W. Zhang, Z.-Y. Zhou, S. Shi, G.-Y. Xiang, X.-S. Wang, Y.-K. Jiang, B.-S. Shi, and G.-C. Guo "Quantum Storage of Orbital Angular Momentum Entanglement in an Atomic Ensemble," Phys. Rev. Lett. 114, 050502 (2015)

[5] M. Gündoğan, P. M. Ledingham, K. Kutluer, M. Mazzera, and H. de Riedmatten "Solid State Spin-Wave Quantum Memory for TimeBin Qubits," Phys. Rev. Lett. 114, 230501 (2015)

[6] D. G. England, K. A. G. Fisher, J.-P. W. MacLean, P. J. Bustard, R. Lausten, K. J. Resch, and B. J. Sussman "Storage and Retrieval of THz-Bandwidth Single Photons Using a Room-Temperature Diamond Quantum Memory," Phys. Rev. Lett. 114, 053602 (2015).

[7] M. D. Eisaman, A. André, F. Massou, M. Fleischhauer, A. S. Zibrov, and M. D. Lukin "Electromagnetically induced transparency with tunable single-photon pulses," Nature 438, 837 (2005) 